\title{
FSI Simulations of Flags Under Varying Wind Conditions
}

\section{Alex Michalski, Raham Zarfam, Ramakrishna Nanjundaiah, Stefan Schöne, Eberhard Haug, Juergen Bradatsch and Rainald Löhner}

\author{
SL Rasch GmbH \\ Special and Lightweight Structures \\ Kesslerweg 22, D-70771 Leinfelden-Echterdingen, Germany \\ e-mail: juergen.bradatsch@sl-rasch.de, web page: https://www.sl-rasch.com/en/
}

\begin{abstract}
The quest for larger and larger flags has required the use of even heavier, more tear-resistant fabrics. On the other hand, the use of these heavier and tougher fabrics should still allow flags to fly in the usual ways under moderate winds in order to display their intended message/colors. In a series of pilot studies, SL-Rasch chose to simulate the behaviour of a tall flag at scale 1:3 to determine the design requirements for the fabric's tensile strength and weight, its attachments, and the flag pole dimensions in order to guarantee the effective and safe performance both at low and high average wind speeds.
\end{abstract}

Simulations of this kind are eminently transient and require the coupling of Computational Fluid Dynamics (CFD) and Computational Structural Dynamics (CSD) codes, i.e. fall under the category of Fluid-Structure Interaction (FSI). The simulations were carried out using PAM-FLOW for the fluid and PAM-CRASH for the membranes, and took into account all the relevant physics (steady and unsteady windfields, linear and nonlinear structural response, contact, load and position transfer, etc.). The FSI simulations made it possible to track the complex waving motions of the flexible fabric in the winds and the stresses in the fabric, as well as the flag pole's bending moments caused by the horizontal drag forces from the flag.

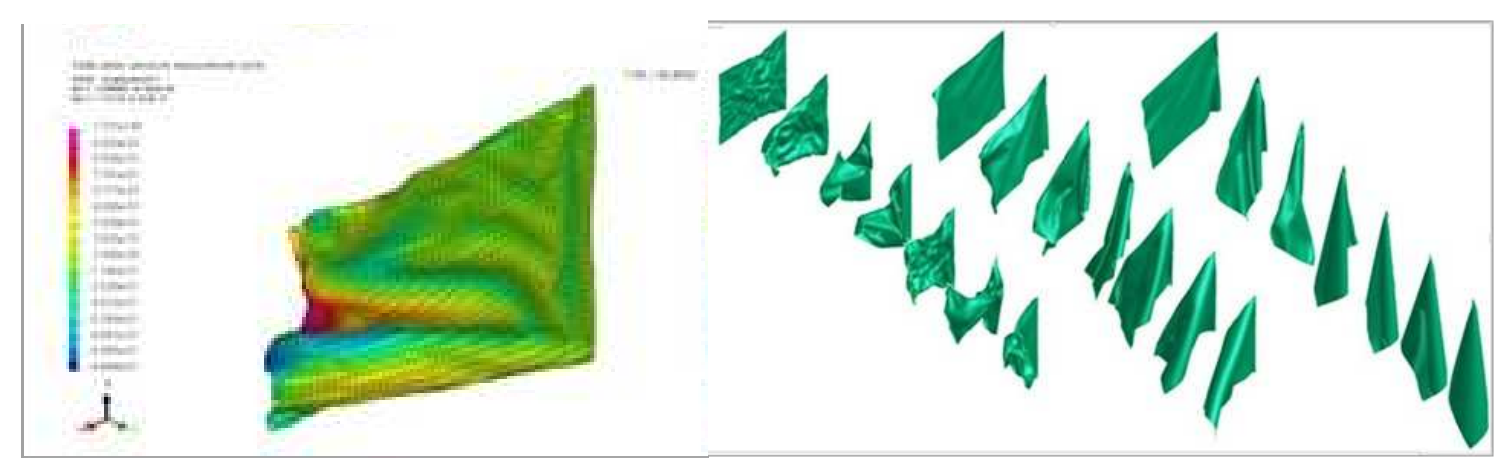

The figure above shows typical output from these runs. An important parameter often sought by flag operators is the lowest average wind speed under which the flag still displays its message. This was determined by a series of simulations that varied (in this case decreased) the wind velocities until the flag was `hanging’ or `sagging’.

The final paper will present a detailed description of the numerical techniques employed, as well as a compilation of the runs carried out and the engineering conclusions drawn from them. 